\title{
Exhaled Breath Analysis of Lung Cancer Patients Using a Metal Oxide Sensor
}

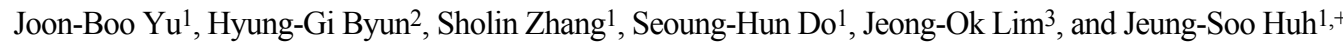

\begin{abstract}
Exhaled breath gases include gases generated in the body. When there is disease in the body, exhalation can include gas components from the disease. If we can find these specific elements through analysis of the exhalation gases, this can be an effective way to diagnose the disease. The lung has a close relationship with exhalation. Lung cancer refers to malignant tumors which originate in the lungs. Exhalation from the lung causes direct jets of gas to be ejected through the mouth and nose, so by analyzing these jets it may be possible to diagnose lung cancer. In our study we attempt to diagnose lung cancer from patient's exhaled gases. Exhalation of lung cancer patients was analyzed using gas chromatography-mass spectroscopy(GC-MS) and the expiratory gas was also measured using a sensor system. The system was designed to use a metal oxide sensor and solid phase micro extraction(SPME) fiber. The GC-MS analysis of the healthy subject's and cancer patient's exhalation gases both showed the presence of decane in the breath of patients with lung cancer. In addition, the results from the sensor system showed significant difference between the lung cancer patients and the healthy subjects.
\end{abstract}

Keywords : GC-MS, Exhaled Breath, Lung Cancer, Disease Diagnosis

\section{INTRODUCTION}

Smell used to be a common diagnostic tool in medicine and physicians were trained to use their sense of smell. In modern times, odor diagnostics has been relegated to a secondary status. Arrays based on gas sensor technology now offer the potential for a robust analytical approach to odor measurement for medical use. This technology has been used to examine odors emitted from breath, wounds, and body fluids to identify possible problems such as gastrointestinal problems, sinus problems, infection, diabetes and liver problems[1-4]. In addition, this technology could be adapted for use inside the human body to detect stomach diseases[5].

Lung cancer accounts for $28 \%$ of cancer-related deaths. Approximately 1.3 million people worldwide die every year for this disease[6]. Breath testing is a fast, noninvasive diagnostic method that links specific volatile organic compounds in exhaled breath to medical conditions[7]. More than 100 compounds have been

\footnotetext{
${ }^{1}$ Department of Material Science and Metallurgy, Kyungpook National University

2 Department of Information \& Communication Engineering, Kangwon National University

${ }^{3}$ Department of Biomedical Science, Kyungpook National University

+ Corresponding author: jshuh@knu.ac.kr

(Received: Jul. 29. 2011, Revised : Sep. 10. 2011, Accepted: Sep. 20. 2011)
}

identified in normal human breath by gas chromatography and mass spectrometry. These volatile organic compounds(VOCs) are produced by metabolic processes and passage from the blood stream via the alveolar pulmonary membrane into alveolar air. This implies that the concentration of these compounds measured in a patient's breath is related to the concentration in their blood. Breath analysis can be used as a diagnostic tool because increased or decreased concentrations of some compounds are associated with various diseases or an altered metabolism. Breath compounds are present in nano molar or even lower quantities. To improve the sensitivity and precision of the detection of these compounds, the sample has to be concentrated before assessment by GCMS. The three main methods currently utilized for preconcentration are chemical interaction, adsorptive binding and cold trapping $[8,9]$.

Here, we wish to develop a screening system for lung cancer. The system for screening lung cancer was made using a metal oxide gas sensor array with solid phase microextraction(SPME) fiber[10, 11]. The system was tested by measuring the breath of lung cancer patients as well as healthy subjects. The breath gas was also analyzed to find the components present by GC-MS. The measurement results of our sensor system were compared with results of the GC-MS analysis. 


\section{EXPERIMENTAL}

\subsection{Collection of exhaled breath}

We collected the exhaled breath of the lung cancer patients and healthy subjects using a tedlar bag. The bag sizes used were $1 \mathrm{~L}$ and $3 \mathrm{~L}$. Exhaled breath sampling was carried out in the morning, before breakfast. After gargling with purified water the gas is collected. The lung cancer patients were selected to be 50 to 75 years old male patients with Stage IIIA and Stage IV cancer. A mouthpiece and aerobic filter for filtering impurities were connected to the tedlar bag while the breath samples were collected.

\subsection{Characteristics of the sensor system}

The sensor system is composed of a metal oxide sensor, a chamber, a data acquisition(DAQ) system, a processor and SPME fiber. The sensor array consisted of a metal oxide sensor with TGS 2600, TGS 2620, TGS 2602 and TGS 2610 by Figaro. The SPME fiber is directly exposed to the breath sample in order to extract and concentrate the breath for analysis. The processor used was a ATmega 64 and the measuring data was saved onto a personal computer. The system including the SPME fiber is shown in Fig. 1. The data processing was performed by principal component analysis(PCA).

\subsection{Chemical analysis of the breath samples}

We identified the VOCs that serve as a biomarker for lung cancer in breath samples and determined their relative composition, using GC-MS(GC: HP5890, MSD: HP5972) in combination with SPME. The column length was $30 \mathrm{~m}$ and the ID was $0.25 \mathrm{~mm}$. The mass range was $29^{\circ} \mathrm{C} \sim 550$ ${ }^{\circ} \mathrm{C}$ The degree of detection was $280^{\circ} \mathrm{C}$ Black fiber SPME was used. The SPME was exposed to the breath sample for 30 minutes, desorption was performed by the GCinjector for five minutes

\subsection{Breath testing with the sensor system}

The SPME fiber was inserted into the tedlar bag and exposed to the collected breath at an ambient temperature of $30{ }^{\circ} \mathrm{C}$ The components of the exhaled gas were allowed to adhere to the SPME fiber for 30 minutes. A chamber was cleaned for 10 minutes with clean air and then the fiber pushed into chamber with the sensor array. The SPME fiber was exposed for 5 minute in the chamber. The SPME fiber was coated with $65 \mu \mathrm{m}$ polydimethylsiloxane /divinylbenzene (PDMS/DVB).

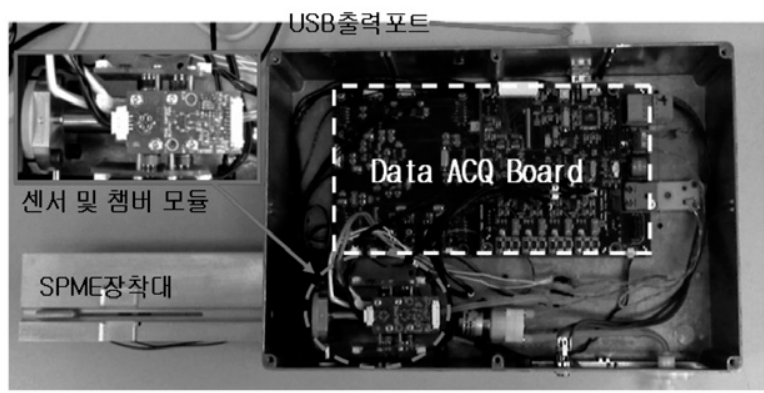

Fig. 1. The measurement sensor system with SPME.

\section{RESULTS AND DISCUSSIONS}

The breath samples results of the patients with lung cancer and healthy subjects are shown in table 1 .

The smokers in the group showed benzene toluene in their sample, while this was not found in non-smoker's samples. The substances which characterized the lung cancer patients were decane, tridecane, octdecane, heneicosane and undecane etc. These components are similar to those found in previous results by other researchers[12].

Table 1. Comparison of Patients with lung cancer and healthy subjects

\begin{tabular}{|c|c|c|c|c|c|c|c|}
\hline \multirow{2}{*}{ Components } & \multicolumn{7}{|c|}{ Area \% } \\
\hline & $\mathrm{S} 1$ & $\mathrm{~S} 2$ & NS1 & NS2 & $\mathrm{P} 1$ & $\mathrm{P} 2$ & P3 \\
\hline Benzene & 0.43 & 0.52 & - & - & - & - & - \\
\hline Toluene & 0.39 & 0.3 & - & - & - & - & - \\
\hline Ethanol & 0.59 & 0.63 & - & 0.71 & 1.36 & 1.1 & 2.97 \\
\hline isoprene & 7.12 & 7.46 & 11.51 & 38.71 & 10.16 & 12.53 & 6.47 \\
\hline pentane & 0.43 & 0.2 & - & - & - & 0.19 & - \\
\hline 2-propanon & 13.52 & 12.04 & 13.48 & 16.35 & 5.57 & 4.42 & 7.61 \\
\hline Heptane & 2.05 & 0.82 & 1.28 & 1.42 & 0.92 & 1.99 & 1.26 \\
\hline Tetradecane & 0.45 & 0.1 & - & - & 0.34 & 0.54 & 0.21 \\
\hline Tridecane & - & - & - & - & 0.32 & 0.54 & 0.41 \\
\hline Octdecane & - & - & - & - & 0.19 & 0.94 & 0.41 \\
\hline Undecane & - & - & - & - & 1.20 & 0.94 & - \\
\hline Heneicosane & - & - & - & - & 0.21 & 0.33 & 0.36 \\
\hline Decane & - & - & - & 0.24 & 1.20 & - & 0.66 \\
\hline
\end{tabular}

$\mathrm{S}$ : Smoker, NS : Non-smoker, P : Patient with lung cancer 

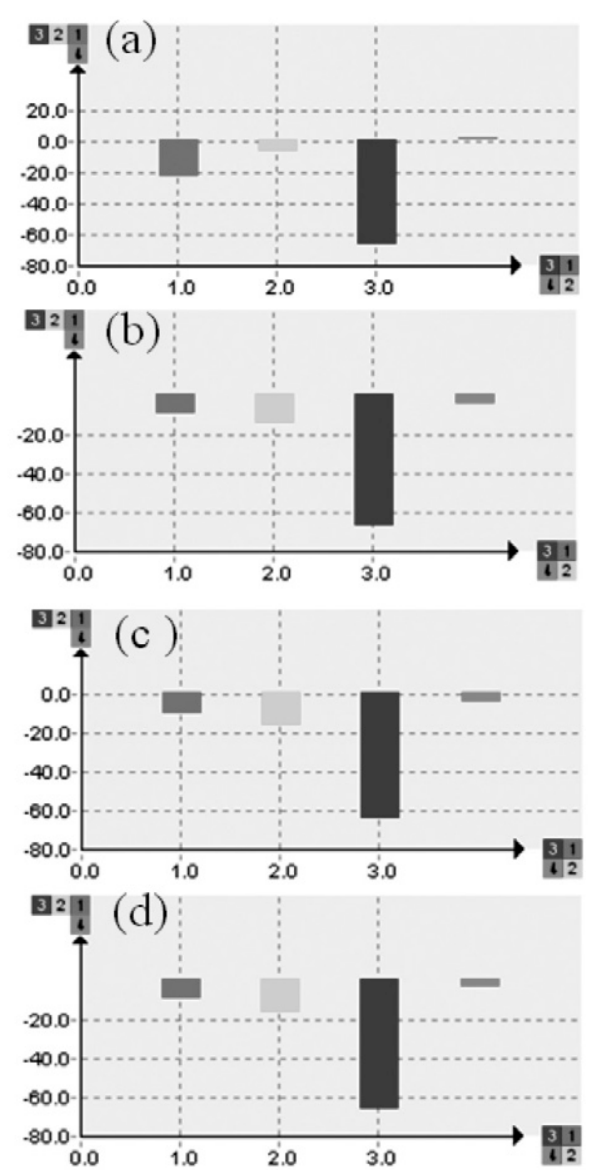

Fig. 2. Test results for healthy subjects and patients with lung cancer (a) 70 year old healthy man (b) 57 years man with lung cancer, smokes (c) 65 years man with lung cancer, smokes (d) 64 years man with lung cancer, non-smoker.

Fig. 2 shows the results for the lung cancer patients and healthy subjects. In both samples, we can clearly distinguish between the subjects with and without lung cancer. The breath of lung cancer patients and healthy subjects were also measured by PCA and the results are shown in Fig. 3.

Breath analysis could be an important element in screening for lung cancer so it is important to properly analyze the breath test results to distinguish between healthy people and lung cancer suffers. Breath testing of preoperative and postoperative lung cancer patients can also be important. Fig. 4 shows the results of a breath test in a 65-year-old cancer patient. Expiratory gas samples were collected before and after surgery within 30 days.

There is not a significant difference in the pattern but the sensitivity of the sensor can be seen from these results. Compared with healthy people, the size of the pattern is estimated to be smaller. Fig. 5 shows the result of the PCA on the breath of lung cancer patients before and after surgery.

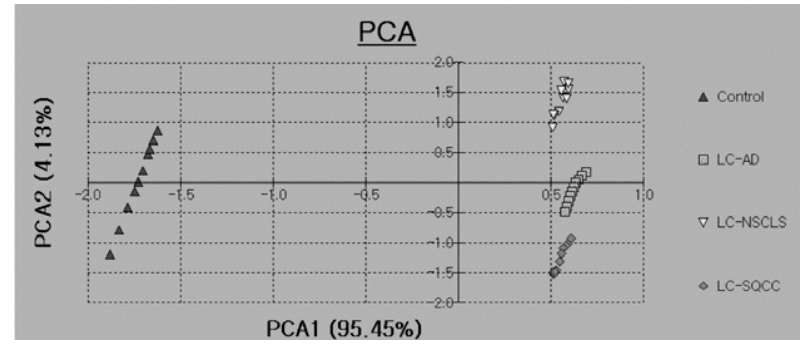

Fig. 3. The principal component analysis of the breath test for the healthy subjects and lung cancer patients; control: healthy subjects, LC-AD: adenocarcinoma, LC-NSCLC: non small cell lung cancer, LC-SQCC: squamous cell carcinoma.
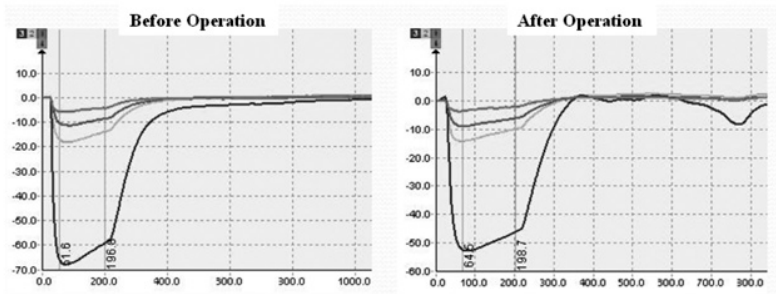

Fig. 4. The response to the breath test of lung cancer pateints before and after surgery.

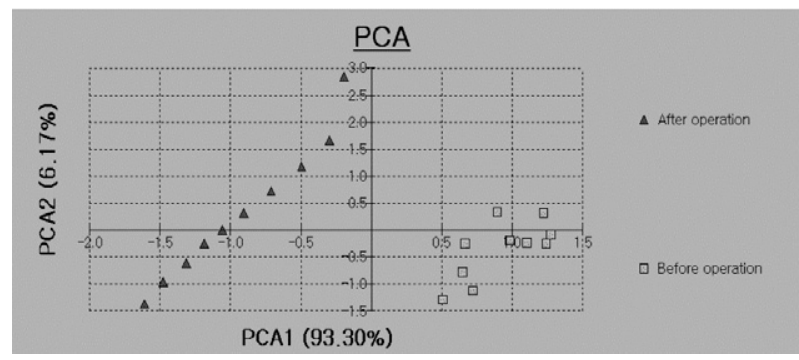

Fig. 5. The principal component analysis of the breath test for lung cancer patients before and after surgery.

\section{CONCLUSIONS}

Breath tests are attractive since they are non-invasive and can be repeated frequently to monitor the changing state of critically ill patients. Analysis of exhaled breath to detect human diseases using endogenous VOCs offers the possibility of noninvasive diagnosis. We investigated a screening method for lung cancer. The system for screening lung cancer was made using a metal oxide gas sensor array with solid phase microextraction(SPME) fiber. The system tested the exhaled gas of lung cancer patients and health subjects. The breath gas was analyzed and the results compared with the components found by GC-MS. 


\section{ACKNOWLEDGMENT}

This research was supported by the Converging Research Center Program funded by the Ministry of Education, Science and Technology (2010K 001133).

\section{REFERENCES}

[1] TD. Gibson, O. Prosser, JN. Hulbert, RW. Marshall, P. Corcoran, P. Lowery, EA. Ruck-Keene, and S. Heron, "Detection and simultaneous identification of micro organisms from headspace samples using electronic nose", Sensors and Actuators B, vol. 44, pp. 413-422, 1997.

[2] J. W. Gardner, H. W. Shin, and E. L. Hines, "An electronic nose system to diagnose illness", Sensors and Actuators B, vol. 70, pp. 19-24, 2000.

[3] J. W. Gardner, R. Dutta, D. Morgan, N. Barker, and E. L. Hines, "Identification of staphlococcus aureus infections in hospital environment: electronic nose based apptoach", Sensors and Actuators B, vol. 109, pp. 355-362, 2005.

[4] J. Yu, H. Byun, M. So, and J. Huh, "Analysis of diabetic patient's breath with conducting polymer sensor array" Sensors and Actuators B, vol. 108, pp. 305-308, 2005.

[5] Y. Lee, K. Song, J. Huh, W. Chung and D. Lee, "Fabrication of clinical gas sensor using MEMS process", Sensors and Actuators B, vol. 108, pp. 292297,2005
[6] G. Peng, U. Tisch, O.Adams, M. Hakim, N. Shehada, Y. Broza, S. Billian, A. Kuten and H Haick, "Diagnosing lung cancer in exhaled breath using gold nanoparticles", Nature nanotechlogy, vol. 4, pp. 669673, 2009.

[7] A. Amann, P. Spanel, and D.Smith, "Breath analysis : the approach towards clinical applications", Mini. Rev. Med. Chem., vol. 7, no. 2, pp. 115-129, 2007.

[8] C. Grote and J. Pawliszyn, "Solid-phase microextraction for the analysis of human breath", Anal. Chem., vol. 69, pp. 587-596, 1997.

[9] W. Cheng and W. Lee, " Technology development in breath micranalysis for clinical daignosis", $J$. Laboratory and Clinnical Medicine, vol. 133, pp. 218228, 1999.

[10] F. D. Cesare, S. Pantalei, E. Zampetti, and A. Macagnano, "Electronic nose and SPME techniques to monitor phenanthrene biodegradation in soil", Sensors and Actuators B, vol. 131, pp. 63-70, 2008.

[11] C. D. Natale, A. Macagnano, E. Martinelli, R. Paolesse, G. D'Arcangelo, C. Roscioni, A. Finazzi, and A. D'Amico, "Lung cancer identification by the analysis of breath by means of an array of nonselective gas sensors", Biosens Bioelectron., vol. 18, pp. 1209-1218., 2003.

[12] M. Phillips, K. Gleeson, J.Michael, B. Huges, J. Greenberg, R. Cataneo, and L.Baker, "Volatile organic compounds in breath as markers of lung cancer: a cross-sectional study", The Lancet, vol. 353, pp. 1930-1933, 1999.

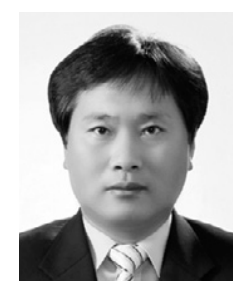

Joon-Boo Yu received the BS, MS degree degree in Electronic Engineering in 1996 from Kwandong University, Korea. In 2010, he received Ph.D. degree in Material Science and Metallurgy from Kyungpook National University, Daegu, Korea. He currently is studying on VOCs monitoring system and chemical sensor for medical application as a researcher in Biomedical Research Institute Kyungpook National University.

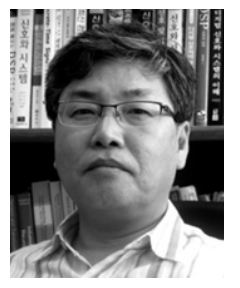

Hyung-Gi Byun received Ph.D. from Manchester University, UK. He is a professor in Department of Information and Communication Engineering from Kangwon National University, Korea. His research is Electronic-nose and Pattern Recognition. 


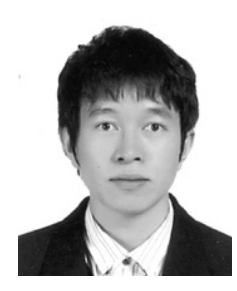

Shaolin Zhang received the BS degree in Materials Science and Engineering from Northwestern Polytechnical University, Xi'an, China, in 2005 and his M.S. degree in Materials Science and Metallurgy from Kyungpook National University, Daegu, Korea in 2007. He received $\mathrm{Ph} . \mathrm{D}$. degree in Sensor and Display Engineering from Kyungpook National University in 2011 with his thesis work concerning controlled synthesis of $\mathrm{ZnO}$ nanostructures and their gas sensor applications. He currently is a Postdoctoral Researcher in Bio-Medical Research Institute in Kyungpook National University Hospital.

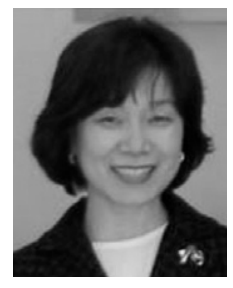

Jeong-Ok Lim received Ph.D. from University of Massachusettts, Lowell, USA. She is a professor in Department of Biomedical Science in Kyungpook National University, Korea. Her research areas include biomaterials and regenerative medicine.

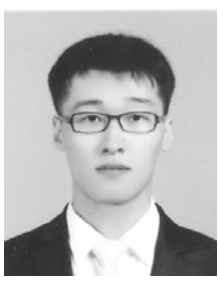

Seoung-Hun Do graduated and received bachelor's degree in Department of Materials Science and Metallurgical Engineering from Kyung-pook National University, Daegu, Korea in 2009, and the M.S. degree in Department of Nano-Science and Technology from Kyung-pook National University, Daegu, Korea in 2011.

Jeung-Soo Huh received Ph.D. from the Department of Materials Science and Engineering (Electronic Materials) in Massachusetts Institute of Technology, USA in 1993. He is directing National Research Lab (Environmental Gas sensor), MOST, Korea. His research areas are chemical/bio sensor and E-nose system. 